\title{
AXISYMMETRIC VIBRATION FOR MICROPOLAR POROUS THERMOELASTIC CIRCULAR PLATE
}

\author{
R. KUMAR ${ }^{*}$ \\ Department of Mathematics \\ Kurukshetra University \\ Kurukshetra, Haryana, INDIA \\ E-mail: rajneesh_kukmath@rediffmail.com \\ P. KAUSHAL \\ Research Scholar (IKG PTU) \\ Kapurthala (Punjab), INDIA \\ E-mail: pkaushal884@gmail.com \\ R. SHARMA \\ Department of Mathematics \\ DAVIET, Jalandhar (Punjab), INDIA \\ E-mail: rajni_daviet@yahoo.com
}

\begin{abstract}
The present investigation is concerned with a two dimensional axisymmetric problem in a homogeneous isotropic micropolar porous thermoelastic circular plate by using the eigen value approach. The Laplace and Hankel transform are used to solve the problem. The expression of displacements, microrotation, volume fraction field, temperature distribution and stresses are obtained in the transformed domain subjected to thermomechanical sources. A computer algorithm is developed for numerical computations. To obtain the resulting quantities in a physical domain, a numerical inversion technique is used. The resulting quantities are depicted graphically for a specific model. Some special cases are also deduced.
\end{abstract}

Key words: micropolar porous thermoelasticity, eigen value, Laplace and Hankel transforms, thermomechanical sources.

\section{Introduction}

The theory of micropolar elasticity developed by Eringen [1] aroused much interest because of its possible utility in investigating the deformation properties of solids for which the classical theory is inadequate. The micropolar theory is significantly useful for investigating materials consisting of bar-like molecules which exhibit microrotation effects and support body and surface couples. A special micropolar material was fabricated in which a uniformly distributed rigid aluminium shot was cast in an elastic epoxy matrix by Gauthier [2] and the values of the relevant parameters based on specimen of aluminium-epoxy composite were investigated.

The linear theory of micropolar thermoelasticity was developed by Eringen [3] and Nowacki [4] to include thermal effects. Touchert et al. [5] developed the linear theory of micropolar thermoelasticity in which Duhamel-Neumann analogy is extended to micropolar materials and the thermoelastic problem is reduced to a corresponding isothermal one with body forces and couples. Boschi and Iesan [6] investigated

\footnotetext{
* To whom correspondence should be addressed
} 
the linear theory of generalized micropolar thermoelasticity. Passeralla [7] established some results in micropolar thermoelasticity.

Cowin and Nunziato [8] investigated the linear theory of elastic materials with voids. This theory differs significantly from the classical linear elasticity in that the volume fraction corresponding to the void volume is taken as an independent kinematical variables. Several applications of the theory are developed, including the response to homogeneous deformations, pure bending of a beam, and small amplitude acoustic waves. In each of these applications, the change in the volume fraction field induced by the deformation is determined. Iesan [9] studied the shock waves in micropolar elastic materials with voids. Iesan [10] developed the linear theory of thermoelastic materials with voids. Scarpetta [11] studied the fundamental solution for the differential system of micropolar elasticity with voids for the steady vibration case and reciprocal properties are also explored. Marin [12] studied the mixed boundary value problem in elastostatic micropolar materials with voids. Marin [13] applied the general results from the theory of elliptic equations to obtain the existence and uniqueness of the generalized solutions for the boundary value problems in micropolar elasticity with voids.

Ciarletta et al. [14] constructed the fundamental solutions of the systems of equations of the steady oscillation of the linear theory of micropolar thermoelasticity for materials with voids. Kumar and Panchal [15] studied the propagation of circular crested waves in a micropolar porous medium possessing cubic symmetry. Ailawalia and Kumar [16] studied the thermomechanical deformation of micropolar generalized thermoelastic materials with voids under the influence of various sources. Othman and Youssef [17] studied the deformation of a micropolar thermoelastic solid with voids considering the influence of various sources acting on the plane interface. Sharma and Marin [18] studied the reflection of plane waves at the free surface of a micropolar generalized thermoelastic solid with distinct conductive and thermodynamic temperatures. Sharma et al. [19] studied the propagation of Lamb waves in a homogeneous, isotropic thermoelastic micropolar solid with two temperatures bordered with layers of inviscid liquid. Sharma [20] studied the effect of two temperatures on the reflection coefficient for a micropolar thermoelastic solid. Sharma and Kumar [21] studied the propagation of plane waves in a thermoviscoelastic medium with voids. Kumar et al. (2015) constructed the fundamental solution to a system of micropolar viscothermoelastic solids with voids in terms of elementary functions. Marin [23] formulated a heat flux dependent theory for micropolar porous materials.

\section{Basic equations}

Following Kumar and Partap [24], the constitutive relations and the field equations in a micropolar porous thermoelastic medium with body forces, body couples, heat sources and extrinsic equilibrated body force are given by

$$
\begin{aligned}
& t_{i j}=\lambda u_{r, r} \delta_{i j}+\mu\left(u_{i, j}+u_{j, i}\right)+K\left(u_{j, i}-\varepsilon_{i j k} \phi_{k}\right)-v\left(1+\tau_{l} \frac{\partial}{\partial t}\right) T \delta_{i j}+b \delta_{i j} \phi^{*}, \\
& m_{i j}=\alpha \phi_{r, r} \delta_{i j}+\beta \phi_{i, j}+\gamma \phi_{j, i}, \\
& (\lambda+\mu) \nabla(\nabla . u)+(\mu+K) \nabla^{2} \boldsymbol{u}+K \nabla \times \phi+b \nabla \phi^{*}-v\left(1+\tau_{l} \frac{\partial}{\partial t}\right) \nabla T=\rho \frac{\partial^{2} \boldsymbol{u}}{\partial t^{2}}, \\
& (\alpha+\beta+\gamma) \nabla(\nabla \cdot \phi)-\gamma \nabla \times(\nabla \times \phi)+K \nabla \times \boldsymbol{u}-2 K \phi=\rho j \frac{\partial^{2} \phi}{\partial t^{2}},
\end{aligned}
$$




$$
\begin{aligned}
& \alpha_{l} \nabla^{2} \phi^{*}-b(\nabla \cdot \boldsymbol{u})-\xi_{l} \phi^{*}-\omega_{0} \frac{\partial \phi^{*}}{\partial t}+m\left(1+\tau_{1} \frac{\partial}{\partial t}\right) T=\rho \chi \frac{\partial^{2} \phi^{*}}{\partial t^{2}} \\
& K_{1}^{*} \nabla^{2} T-v \mathrm{~T}_{0}\left(\frac{\partial}{\partial t}+\eta_{0} \tau_{0} \frac{\partial^{2}}{\partial t^{2}}\right)(\nabla \cdot \boldsymbol{u})-\mathrm{mT}_{0}\left(\frac{\partial}{\partial t}+\eta_{0} \tau_{0} \frac{\partial^{2}}{\partial t^{2}}\right) \phi^{*}=\rho C^{*}\left(\frac{\partial}{\partial t}+\tau_{0} \frac{\partial^{2}}{\partial t^{2}}\right) T
\end{aligned}
$$

where $\boldsymbol{u}$ is the displacement vector, $\boldsymbol{\phi}$ is the microrotation vector, $\rho$ is the density, $j$ is the micro inertia, $K_{1}^{*}$ is the coefficient of thermal conductivity, $T$ is the change in temperature of the medium at any time, $C^{*}$ is the specific heat at constant strain, $\phi^{*}$ is the change in volume fraction field, $\lambda, \mu, K, \alpha, \beta, \gamma$ are micropolar constants, $\alpha_{1}, b, \xi_{1}, \omega_{0}, m$ and $\chi$ are the elastic constants due to the presence of voids, $v=(3 \lambda+2 \mu+K) \alpha_{t}, \alpha_{t}$ is the coefficient of linear thermal expansion, $\tau_{0}, \tau_{l}$, are the thermal relaxation times, $t_{i j}, m_{i j}$ are the stress tensor and couple stress tensor, $\delta_{i j}$ is the Kronecker delta and $\nabla^{2}=\frac{\partial^{2}}{\partial r^{2}}+\frac{1}{r} \frac{\partial}{\partial \mathrm{r}}+\frac{1}{r^{2}} \frac{\partial^{2}}{\partial \theta^{2}}+\frac{\partial^{2}}{\partial z^{2}}$ is the Laplacian operator. For L-S theory, $\tau_{1}=0, \tau_{0}>0$, and $\eta_{0}=1$. For G-L theory, $\tau_{1} \geq \tau_{0}>0$ and $\eta_{0}=0$.

\section{Formulation of the problem}

Consider an infinite, homogeneous isotropic micropolar porous thermoelastic circular plate of thickness $2 h$ occupying the region defined by $0 \leq r \leq \infty,-h \leq z \leq h$. The cylindrical polar coordinates $(r, \theta, z)$ are introduced. The plate is axisymmetric with the $z$-axis as the axis of symmetry. The origin of the co- ordinate system $(r, \theta, z)$ is taken as the middle surface of the plate and the $z$-axis normal to it along the thickness. We take the $r-z$ plane as the plane of incidence. The initial temperature in the thick plate is given by a constant temperature $T_{0}$.

For a two dimensional problem, we take

$$
\boldsymbol{u}=\left(u_{r}, 0, u_{z}\right), \quad \phi=\left(0, \phi_{\theta}, 0\right) .
$$

The dimensionless quantities are given by

$$
\begin{aligned}
& r^{\prime}=\frac{\omega^{*} r}{c_{1}}, \quad z^{\prime}=\frac{\omega^{*} z}{c_{1}}, \quad u_{r}^{\prime}=\frac{\rho c_{1} \omega^{*} u_{r}}{v T_{0}}, \quad u_{z}^{\prime}=\frac{\rho c_{1} \omega^{*} u_{z}}{v T_{0}}, \quad \phi_{\theta}^{\prime}=\frac{\rho c_{1}^{2} \phi_{\theta}}{v T_{0}}, \quad \phi^{* \prime}=\frac{\rho c_{1}^{2} \phi^{*}}{v T_{0}}, \\
& T^{\prime}=\frac{T}{T_{0}}, \quad t^{\prime}=\omega^{*} t, \quad \tau_{0}^{\prime}=\omega^{*} \tau_{0}, \quad \tau_{1}^{\prime}=\omega^{*} \tau_{1}, \quad t_{i j}^{\prime}=\frac{t_{i j}}{v T_{0}}, \quad m_{i j}^{\prime}=\frac{\omega^{*}}{c_{1} v T_{0}} m_{i j}
\end{aligned}
$$

where

$$
c_{1}^{2}=\frac{\lambda+2 \mu+K}{\rho}, \quad \omega^{*}=\frac{K}{\rho j} .
$$

We define the Laplace and Hankel transform as 


$$
\begin{aligned}
& \bar{f}(r, z, s)=L\{\bar{f}(r, z, t)\}=\int_{0}^{\infty} f(r, z, t) e^{-s t} d t, \\
& \tilde{f}(\xi, z, s)=H\{\bar{f}(x, z, s)\}=\int_{0}^{\infty} r \bar{f}(x, z, s) J_{n}(\xi r) d r .
\end{aligned}
$$

Equations (2.1)-(2.4) with the aid (3.1)-(3.4) recast into the form

$$
\begin{aligned}
& \tilde{u}_{r}^{\prime \prime}=a_{11} \tilde{u}_{r}+a_{14} \phi^{*}+a_{15} \tilde{T}+b_{12} \tilde{u}_{z}^{\prime}+b_{13} \tilde{\phi}_{\theta}^{\prime}, \\
& \tilde{u}_{z}^{\prime \prime}=a_{22} \tilde{u}_{z}+a_{23} \tilde{\phi}_{\theta}+b_{21} \tilde{u}_{r}^{\prime}+b_{24} \tilde{\phi}^{* \prime}+b_{25} \tilde{T}^{\prime}, \\
& \tilde{\phi}_{\theta}^{\prime \prime}=a_{32} \tilde{u}_{z}+a_{33} \tilde{\phi}_{\theta}+b_{31} \tilde{u}_{r}^{\prime}, \\
& \tilde{\phi}^{* \prime \prime}=a_{41} \tilde{u}_{r}+a_{44} \tilde{\phi}^{*}+a_{45} \tilde{T}+b_{42} \tilde{u}_{z}^{\prime}, \\
& \tilde{T}^{\prime \prime}=a_{51} \tilde{u}_{r}+a_{54} \tilde{\phi}^{*}+a_{55} \tilde{T}+b_{52} \tilde{u}_{z}^{\prime}
\end{aligned}
$$

where

$$
\begin{aligned}
& a_{11}=\left(\frac{\xi^{2}+s^{2}}{\delta^{2}}\right), \quad a_{14}=\frac{p_{0} \xi}{\delta^{2}}, \quad a_{15}=-\frac{\xi}{\delta^{2}}\left(1+\tau_{1} s\right), \quad a_{22}=\left(\xi^{2} \delta^{2}+s^{2}\right), \quad a_{23}=-p \xi, \\
& a_{32}=-\xi \delta^{* 2}, \quad a_{33}=\left(\xi^{2}+\frac{s^{2}}{\delta_{1}^{2}}+2 \delta^{* 2}\right), \quad a_{41}=p_{0} \delta_{1}^{*} \xi, \quad a_{44}=\left(\xi^{2}+\delta_{3}^{*} s^{2}+p_{1} \delta_{1}^{*}+\delta_{2}^{*} s\right), \\
& a_{45}=-\bar{v} \delta_{1}^{*}\left(1+\tau_{1} s\right), \quad a_{51}=\xi \epsilon\left(s+\eta_{0} \tau_{0} s^{2}\right), \quad a_{54}=\bar{v} \epsilon\left(s+\eta_{0} \tau_{0} s^{2}\right), \\
& a_{55}=\left(\xi^{2}+Q^{*}\left(s+\tau_{0} s^{2}\right)\right), \quad b_{12}=\frac{\xi\left(1-\delta^{2}\right)}{\delta^{2}}, \quad b_{13}=\frac{p}{\delta^{2}}, \quad b_{21}=-\xi\left(1-\delta^{2}\right), \\
& b_{24}=-p_{0}, \quad b_{25}=\left(1+\tau_{1} s\right), \quad b_{31}=-\delta^{* 2}, \quad b_{42}=p_{0} \delta_{1}^{*}, \quad b_{52}=\epsilon\left(s+\eta_{0} \tau_{0} s^{2}\right), \\
& c_{2}^{2}=\frac{\mu+K}{\rho}, \quad \delta^{2}=\frac{c_{2}^{2}}{c_{1}^{2}}, \quad p=\frac{K}{\rho c_{1}^{2}}, \quad p_{0}=\frac{b}{\rho c_{1}^{2}}, \quad \delta^{* 2}=\frac{K c_{1}^{2}}{\gamma \omega^{* 2}}, \quad \delta_{1}^{2}=\frac{c_{3}^{2}}{c_{1}^{2}}, \\
& Q^{*}=\frac{\rho C^{*} c_{1}^{2}}{K_{1}^{*} \omega^{*}}, \quad \epsilon=\frac{v^{2} T_{0}}{\rho K_{1}^{*} \omega^{*}} . \quad \xi_{1}^{2}=\frac{\gamma}{\rho j}, \quad \delta_{1}^{*}=\frac{\rho c_{1}^{4}}{\alpha_{1} \omega^{* 2}}, \quad \bar{v}=\frac{m}{v}, \quad p_{1}=\frac{\xi_{1}}{\rho c_{1}^{2}}, \quad \delta_{2}^{*}=\frac{\omega_{0} c_{1}^{2}}{\alpha_{1} \omega^{*}, \quad \delta_{3}^{*}=\frac{\rho \chi c_{1}^{2}}{\alpha_{1}},}
\end{aligned}
$$


The system of Eqs (3.5)-(3.9) can be written as

$$
\frac{d}{d z} W(\xi, z, s)=A(\xi, s) W(\xi, z, s)
$$

where

$$
\begin{aligned}
W & =\left[\begin{array}{c}
U \\
D U
\end{array}\right], \quad A=\left[\begin{array}{cc}
O & I \\
A_{2} & A_{1}
\end{array}\right], \quad U=\left[\begin{array}{c}
\tilde{u} \\
u_{r} \\
\tilde{u}_{z} \\
\tilde{\phi}_{\theta} \\
\tilde{\phi}^{*} \\
\tilde{T}
\end{array}\right], \quad D=\frac{d}{d z}, \\
O & =\left[\begin{array}{ccccc}
0 & 0 & 0 & 0 & 0 \\
0 & 0 & 0 & 0 & 0 \\
0 & 0 & 0 & 0 & 0 \\
0 & 0 & 0 & 0 & 0 \\
0 & 0 & 0 & 0 & 0
\end{array}\right], \quad I=\left[\begin{array}{ccccc}
1 & 0 & 0 & 0 & 0 \\
0 & 1 & 0 & 0 & 0 \\
0 & 0 & 1 & 0 & 0 \\
0 & 0 & 0 & 1 & 0 \\
0 & 0 & 0 & 0 & 1
\end{array}\right], \\
A_{2} & \left.=\left[\begin{array}{ccccc}
a_{11} & 0 & 0 & a_{14} & a_{15} \\
0 & a_{22} & a_{23} & 0 & 0 \\
0 & a_{32} & a_{33} & 0 & 0 \\
a_{41} & 0 & 0 & a_{44} & a_{45} \\
a_{51} & 0 & 0 & a_{54} & a_{55}
\end{array}\right], \quad \begin{array}{ccccc}
0 & b_{12} & b_{13} & 0 & 0 \\
b_{21} & 0 & 0 & b_{24} & b_{25} \\
b_{31} & 0 & 0 & 0 & 0 \\
0 & b_{42} & 0 & 0 & 0 \\
0 & b_{52} & 0 & 0 & 0
\end{array}\right] .
\end{aligned}
$$

To solve Eq.(3.10), we take

$$
W(\xi, z, s)=X(\xi, s) e^{q z}
$$

with

$$
A(\xi, s) W(\xi, z, s)=q W(\xi, z, s)
$$

which leads to the eigen value problem. The characteristic equation corresponding to Eq.(3.13) on expansion, yields

$$
q^{10}-\lambda_{1} q^{8}+\lambda_{2} q^{6}-\lambda_{3} q^{4}+\lambda_{4} q^{2}-\lambda_{5}=0
$$

where $\lambda_{1}, \lambda_{2}, \lambda_{3}, \lambda_{4}$ and $\lambda_{5}$ are given in Appendix I and $\pm q_{i},(i=1,2,3,4,5)$ are the roots of Eq.(3.14).

The eigenvectors $X_{i}(\xi, s)$ corresponding to the eigenvalues $q_{i}$ may be obtained by solving

$$
[A-q I] X_{i}(\xi, s)=0
$$


The set of eigen vector $X_{i}(\xi, s)$ can be written as

$$
X_{i}(\xi, s)=\left[\begin{array}{c}
X_{i 1}(\xi, s) \\
X_{i 2}(\xi, s)
\end{array}\right]
$$

where

$$
\begin{aligned}
X_{i 1}(\xi, s) & =\left[\begin{array}{c}
a_{i} q_{i} \\
b_{i} \\
-\xi \\
d_{i} \\
e_{i}
\end{array}\right], \quad X_{i 2}(\xi, s)=\left[\begin{array}{c}
a_{i} q_{i}^{2} \\
b_{i} q_{i} \\
-\xi q_{i} \\
d_{i} q_{i} \\
e_{i} q_{i}
\end{array}\right], \quad q=q_{i} ; i=1,2,3,4,5, \\
X_{j 1}(\xi, s) & =\left[\begin{array}{c}
-a_{i} q_{i} \\
b_{i} \\
-\xi \\
d_{i} \\
e_{i}
\end{array}\right], \quad X_{j 2}(\xi, s)=\left[\begin{array}{c}
a_{i} q_{i}^{2} \\
-b_{i} q_{i} \\
\xi q_{i} \\
-d_{i} q_{i} \\
-e_{i} q_{i}
\end{array}\right], \quad j=i+5, q=-q_{i} ; i=1,2,3,4,5
\end{aligned}
$$

Where $a_{i}, b_{i}, d_{i}, e_{i}, \Delta_{i}, r_{1}, r_{2}, r_{3}, r_{4}$ and $r_{5}$ are given in Appendix II

We assume the solution of Eq.(3.12) as

$$
W(\xi, z, s)=\sum_{i=1}^{5} K_{i} X_{i}(\xi, s) \cosh \left(q_{i} z\right)
$$

where $K_{1}, K_{2}, K_{3}, K_{4}$ and $K_{5}$ are arbitrary constants.

\section{Boundary conditions}

The boundary conditions at the surface $z= \pm h$ of the plate are given by

$$
\begin{aligned}
& \frac{d T}{d z}=z^{2} H(a-r), \\
& t_{z z}=p_{0} \frac{\delta(r) H(t)}{2 \pi r}, \\
& t_{z r}=0 \\
& m_{z \theta}=0
\end{aligned}
$$




$$
\frac{d \phi^{*}}{d z}=0
$$

$(\delta)$ is a Dirac delta function and $(H)$ is the Heavy side unit step function and $t_{z z}, t_{z r}$ and $m_{z \theta}$ are given by

$$
\begin{aligned}
& t_{z z}=(\lambda+2 \mu+K) \frac{\partial u_{z}}{\partial z}+\lambda\left(\frac{\partial u_{r}}{\partial r}+\frac{u_{r}}{r}\right)-v\left(1+\tau_{1} \frac{\partial}{\partial r}\right) T+b \phi^{*}, \\
& t_{z r}=(\mu+K) \frac{\partial u_{r}}{\partial z}+\mu \frac{\partial u_{z}}{\partial r}-K \phi_{\theta}, \\
& m_{z \theta}=\gamma \frac{\partial \phi_{\theta}}{\partial z} .
\end{aligned}
$$

From Eqs (3.2)-(3.4) and (3.15)-(4.8), after some algebraic calculations, we get

$$
\begin{aligned}
& \left(\tilde{u}_{r}, \tilde{u}_{z}, \tilde{\phi}_{\theta}, \tilde{\phi}^{*}, \tilde{T}\right)=\frac{1}{\Delta} \sum_{i=1}^{5}\left(a_{i} q_{i}, b_{i},-\xi, d_{i}, e_{i}\right) \Delta_{i} \cosh \left(q_{i} z\right), \\
& \left(\tilde{t}_{z z}, \tilde{t}_{z r}, \tilde{m}_{z \theta}\right)=\frac{1}{\Delta} \sum_{i=1}^{5}\left(L_{i}, M_{i}, P_{i}\right) \Delta_{i} \cosh \left(q_{i} z\right)
\end{aligned}
$$

where

$$
\Delta=\left|\begin{array}{ccccc}
S_{1} & S_{2} & S_{3} & S_{4} & S_{5} \\
T_{1} & T_{2} & T_{3} & T_{4} & T_{5} \\
U_{1} & U_{2} & U_{3} & U_{4} & U_{5} \\
V_{1} & V_{2} & V_{3} & V_{4} & V_{5} \\
W_{1} & W_{2} & W_{3} & W_{4} & W_{5}
\end{array}\right|,
$$

and $\Delta_{i}(i=1,2,3,4,5)$ are obtained from $\Delta$ by replacing $\mathrm{i}^{\text {th }}$ column of $\Delta$ with $|Q, R, 0,0, O|^{t r}$, also $S_{i}=e_{i} q_{i} \cosh \left(q_{i} h\right), T_{i}=L_{i} \cosh \left(q_{i} h\right), U_{i}=M_{i} \cosh \left(q_{i} h\right), \quad V_{i}=P_{i} \cosh \left(q_{i} h\right)$,

$$
\begin{aligned}
& W_{i}=d_{i} q_{i} \cosh \left(q_{i} h\right), \quad Q=\frac{a z^{2} J_{l}(\xi a)}{\xi}, \quad R=\frac{p_{0}}{2 \pi s}, \\
& L_{i}=\left[\frac{\lambda \xi a_{i} q_{i}}{\rho c_{1}^{2}}+p_{0} d_{i}-\left(1+\tau_{l} s\right) e_{i}+\left(\frac{\lambda+2 \mu+K}{\rho c_{1}^{2}}\right) b_{i} q_{i}\right], \quad i=1,2,3,4,5, \\
& M_{i}=\left[-\frac{\mu \xi b_{i}}{\rho c_{1}^{2}}+\frac{\xi K}{\rho c_{1}^{2}}+\left(\frac{\mu+K}{\rho c_{1}^{2}}\right) a_{i} q_{i}^{2}\right], \quad i=1,2,3,4,5,
\end{aligned}
$$




$$
P_{i}=\frac{-\gamma \xi \omega^{* 2} q_{i}}{\rho c_{1}^{4}}, \quad i=1,2,3,4,5
$$

\section{Particular cases}

(i) Without the thermal effect, the boundary conditions (4.1)-(4.5) for a micropolar porous medium reduce to the form

$$
t_{z z}=p_{0} \frac{\delta(r) H(t)}{2 \pi r}, \quad t_{z r}=0, \quad m_{z \theta}=0, \quad \frac{d \phi^{*}}{d z}=0
$$

and following the same procedure, the corresponding expressions for displacements, microrotation, volume fraction field and stresses for a micropolar porous are obtained as

$$
\begin{aligned}
& \left(\tilde{u}_{r}, \tilde{u}_{z}, \tilde{\phi}_{\theta}, \tilde{\phi}^{*}\right)=\frac{1}{\Delta^{*}} \sum_{i=1}^{4}\left(a_{i} q_{i}, b_{i},-\xi, d_{i}\right) \Delta_{i}^{*} \cosh \left(q_{i} z\right), \\
& \left(\tilde{t}_{z z}, \tilde{t}_{z r}, \tilde{m}_{z \theta}\right)=\frac{1}{\Delta^{*}} \sum_{i=1}^{4}\left(L_{i}, M_{i}, P_{i}\right) \Delta_{i}^{*} \cosh \left(q_{i} z\right)
\end{aligned}
$$

where

$$
\Delta^{*}=\left|\begin{array}{cccc}
T_{1}^{*} & T_{2}^{*} & T_{3}^{*} & T_{4}^{*} \\
U_{1}^{*} & U_{2}^{*} & U_{3}^{*} & U_{4}^{*} \\
V_{1}^{*} & V_{2}^{*} & V_{3}^{*} & V_{4}^{*} \\
W_{1}^{*} & W_{2}^{*} & W_{3}^{*} & W_{4}^{*}
\end{array}\right|,
$$

and $\Delta_{i}^{*}(i=1,2,3,4)$ are obtained from $\Delta^{*}$ by replacing $\mathrm{i}^{\text {th }}$ column of $\Delta^{*}$ with $|R, 0,0, O|^{t r}$, also

$$
\begin{aligned}
& T_{i}^{*}=L_{i}^{*} \cosh \left(q_{i} h\right), \quad U_{i}^{*}=M_{i}^{*} \cosh \left(q_{i} h\right), \quad V_{i}^{*}=P_{i}^{*} \cosh \left(q_{i} h\right), \quad W_{i}^{*}=d_{i} q_{i} \cosh \left(q_{i} h\right), \\
& L_{i}^{*}=\left[\frac{\lambda \xi a_{i} q_{i}}{\rho c_{1}^{2}}+p_{0} d_{i}+\left(\frac{\lambda+2 \mu+K}{\rho c_{1}^{2}}\right) b_{i} q_{i}\right], \quad i=1,2,3,4, \\
& M_{i}^{*}=\left[-\frac{\mu \xi b_{i}}{\rho c_{1}^{2}}+\frac{\xi K}{\rho c_{1}^{2}}+\left(\frac{\mu+K}{\rho c_{1}^{2}}\right) a_{i} q_{i}^{2}\right], \quad i=1,2,3,4, \\
& P_{i}^{*}=\frac{-\gamma \xi \omega^{* 2} q_{i}}{\rho c_{1}^{4}}, \quad i=1,2,3,4 .
\end{aligned}
$$

(ii) Neglecting the porous effect, i.e., $\alpha_{1}, b, \xi_{1}, \omega_{0}, \chi$ and $\phi^{*} \rightarrow 0$, yields the boundary conditions for micropolar thermoelastic medium as 


$$
\frac{d T}{d z}=z^{2} H(a-r), \quad t_{z z}=p_{0} \frac{\delta(r) H(t)}{2 \pi r}, \quad t_{z r}=0, \quad m_{z \theta}=0
$$

and corresponding expressions are given by

$$
\begin{aligned}
& \left(\tilde{u}_{r}, \tilde{u}_{z}, \tilde{\phi}_{\theta}, \tilde{T}\right)=\frac{1}{\Delta^{* *}} \sum_{i=1}^{4}\left(a_{i} q_{i}, b_{i},-\xi, e_{i}\right) \Delta_{i}^{* *} \cosh \left(q_{i} z\right), \\
& \left(\tilde{t}_{z z}, \tilde{t}_{z r}, \tilde{m}_{z \theta}\right)=\frac{1}{\Delta^{* *}} \sum_{i=1}^{4}\left(L_{i}, M_{i}, P_{i}\right) \Delta_{i}^{* *} \cosh \left(q_{i} z\right)
\end{aligned}
$$

where

$$
\Delta^{* *}=\left|\begin{array}{cccc}
S_{1}^{* *} & S_{2}^{* *} & S_{3}^{* *} & S_{4}^{* *} \\
T_{1}^{* *} & T_{2}^{* *} & T_{3}^{* *} & T_{4}^{* *} \\
U_{1}^{* *} & U_{2}^{* *} & U_{3}^{* *} & U_{4}^{* *} \\
V_{1}^{* *} & V_{2}^{* *} & V_{3}^{* *} & V_{4}^{* *}
\end{array}\right|,
$$

and $\Delta_{i}^{* *}(i=1,2,3,4)$ are obtained from $\Delta^{* *}$ by replacing $\mathrm{i}^{\text {th }}$ column of $\Delta^{* *}$ with $|Q, R, 0,0|^{t r}$, also

$$
\begin{aligned}
& S_{i}^{* *}=e_{i} q_{i} \cosh \left(q_{i} h\right), \quad T_{i}^{* *}=L_{i}^{* *} \cosh \left(q_{i} h\right), \quad U_{i}^{* *}=M_{i}^{* *} \cosh \left(q_{i} h\right), \quad V_{i}^{* *}=P_{i}^{* *} \cosh \left(q_{i} h\right), \\
& L_{i}^{* *}=\left[\frac{\lambda \xi a_{i} q_{i}}{\rho c_{1}^{2}}-\left(1+\tau_{1} s\right) e_{i}+\left(\frac{\lambda+2 \mu+K}{\rho c_{1}^{2}}\right) b_{i} q_{i}\right], \quad i=1,2,3,4 \\
& M_{i}^{* *}=\left[-\frac{\mu \xi b_{i}}{\rho c_{1}^{2}}+\frac{\xi K}{\rho c_{1}^{2}}+\left(\frac{\mu+K}{\rho c_{1}^{2}}\right) a_{i} q_{i}^{2}\right], \quad i=1,2,3,4, \\
& P_{i}^{* *}=\frac{-\gamma \xi \omega^{* 2} q_{i}}{\rho c_{1}^{4}}, \quad i=1,2,3,4 .
\end{aligned}
$$

(iii) Taking $\tau_{1}=0, \eta_{0}=1$, in Eqs (4.9)-(4.10), yields the corresponding expressions for micropolar porous thermoelastic with one relaxation time.

(iv) The corresponding expressions for a micropolar porous thermoelastic with two relaxation times are obtained by taking $\tau_{1}>0, \eta_{0}=0$ in Eqs (4.9)-(4.10).

\section{Inversion of transforms}

The transformed displacements, microrotation vector, volume fraction field, temperature distribution and stresses are of the form $\tilde{f}(\xi, z, s)$ and to obtain the function $f(r, z, t)$, the inversion of the Hankel transform is of the form 


$$
\tilde{f}(\xi, z, s)=\int_{0}^{\infty} \xi \bar{f}(\xi, z, s) J_{n}(\xi r) d \xi .
$$

The inversion formula for the Laplace transforms is given as

$$
f(r, z, t)=\frac{1}{2 \imath \pi} \int_{c-1 \infty}^{c+1 \infty} \bar{f}(r, z, s) e^{-s t} d s
$$

where $c$ is an arbitrary constant greater than all real parts of the singularities of $\bar{f}(r, z, t)$.

\section{Numerical results and discussion}

The values of micropolar parameters for numerical computations are given by Eringen [25]

$$
\begin{array}{ll}
\lambda=9.4 \times 10^{10} \mathrm{Nm}^{-2}, \quad \mu=4.0 \times 10^{10} \mathrm{Nm}^{-2}, & K=1.0 \times 10^{10} \mathrm{Nm}^{-2}, \\
\rho=1.74 \times 10^{3} \mathrm{Kgm}^{-3}, & j=0.2 \times 10^{-19} \mathrm{~m}^{2},
\end{array}
$$

Following Dhaliwal and Singh [26], we take the values of thermal parameters as

$$
\begin{aligned}
& C^{*}=1.04 \times 10^{3} \mathrm{JKg}^{-1} \mathrm{~K}^{-1}, \quad K_{1}^{*}=1.7 \times 10^{6} \mathrm{Jm}^{-1} \mathrm{~s}^{-1} \mathrm{~K}^{-1}, \alpha_{t}=2.33 \times 10^{-5} \mathrm{~K}^{-1}, \\
& \tau_{0}=6.131 \times 10^{-13} \mathrm{sec}, \quad \tau_{l}=8.765 \times 10^{-13} \mathrm{sec}, \quad m=1.13849 \times 10^{10} \mathrm{~N} / \mathrm{m}^{2}, \\
& T_{0}=0.298 \times 10^{3} \mathrm{~K} .
\end{aligned}
$$

The values of void parameters are taken as

$$
\begin{aligned}
& \alpha_{1}=3.688 \times 10^{-9} \mathrm{~N}, \quad b=1.138494 \times 10^{10} \mathrm{~N} / \mathrm{m}^{2}, \quad \xi_{1}=1.1475 \times 10^{10} \mathrm{~N} / \mathrm{m}^{2}, \\
& \chi=1.1753 \times 10^{-19} \mathrm{~m}^{2}, \quad \omega_{0}=0.0787 \times 10^{-1} \mathrm{~N} \times \mathrm{sec} / \mathrm{m}^{2} .
\end{aligned}
$$

The variations of displacements, microrotation, volume fraction field, temperature distribution and stresses with distance $r$ in the case of a micropolar thermoelastic porous medium (MTPM), micropolar thermoelastic medium (MTM) and micropolar porous medium (MPM) are shown in Figs 1-8, respectively. In all these figures, a solid line (- $)$, a small dash line (- - - ) and a dash line with centred symbol $(-\cdot-\cdot)$ are used for MTPM, MPM and MTM, respectively. 


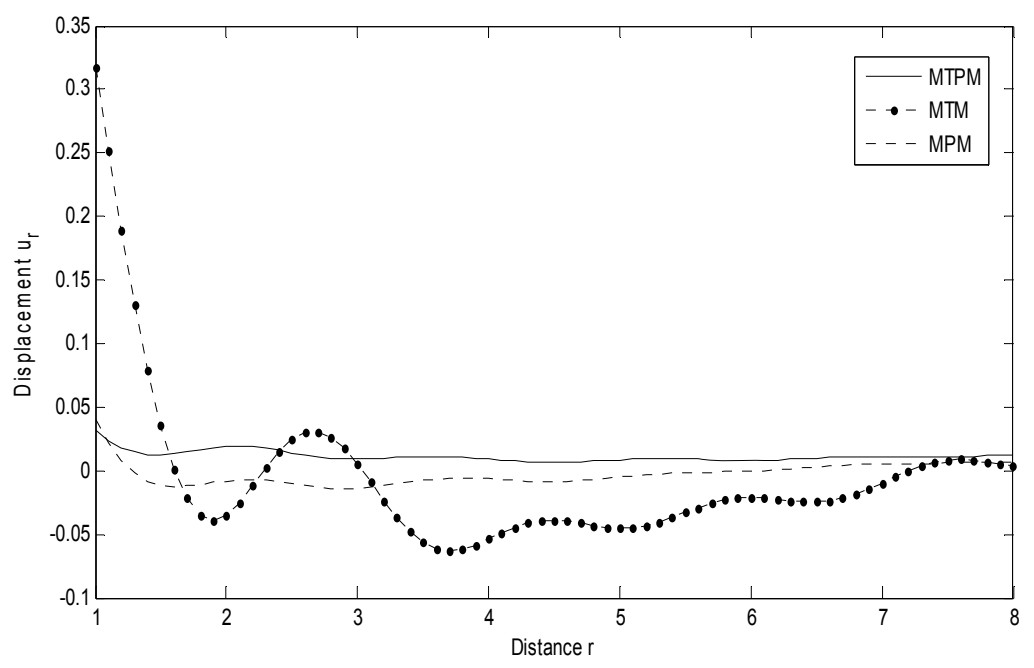

Fig.1. Variations of displacement of $u_{r}$.

Figure 1 depicts that the value of $u_{r}$ initially decreases for $1 \leq r \leq 1.8$ and then oscillates for $1.8 \leq r \leq 8$ with large amplitude for MTPM, MTM and MPM. For MTM, $u_{r}$ has a maximum value at the beginning and a minimum value for $3.4 \leq r \leq 7.4$ in comparison with MTPM and MPM. The oscillation behavior is not uniform for the three cases.

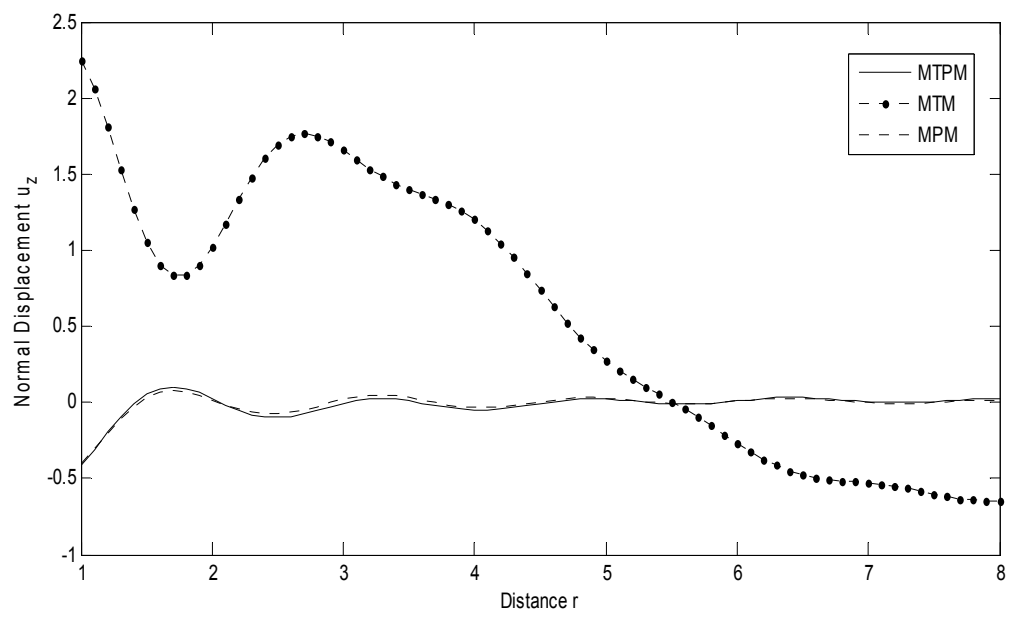

Fig.2. Variations of normal displacement $u_{z}$.

Figure 2 shows that the value of $u_{z}$ which initially increases for $1 \leq r \leq 1.8$ and then oscillates for the whole range with large amplitude for MTPM and MPM. The value of $u_{z}$ for MTM decreases for $1 \leq r \leq 1.7$, increases for $1.7 \leq r \leq 2.8$ and then rapidly decreases for $2.8 \leq r \leq 8$. The variation and behavior for MTPM and MPM are similar with a slightly different magnitude. The values of normal displacement for MTPM and MPM are small compared to MTM for $1 \leq r \leq 5.6$ and the values approach zero. 


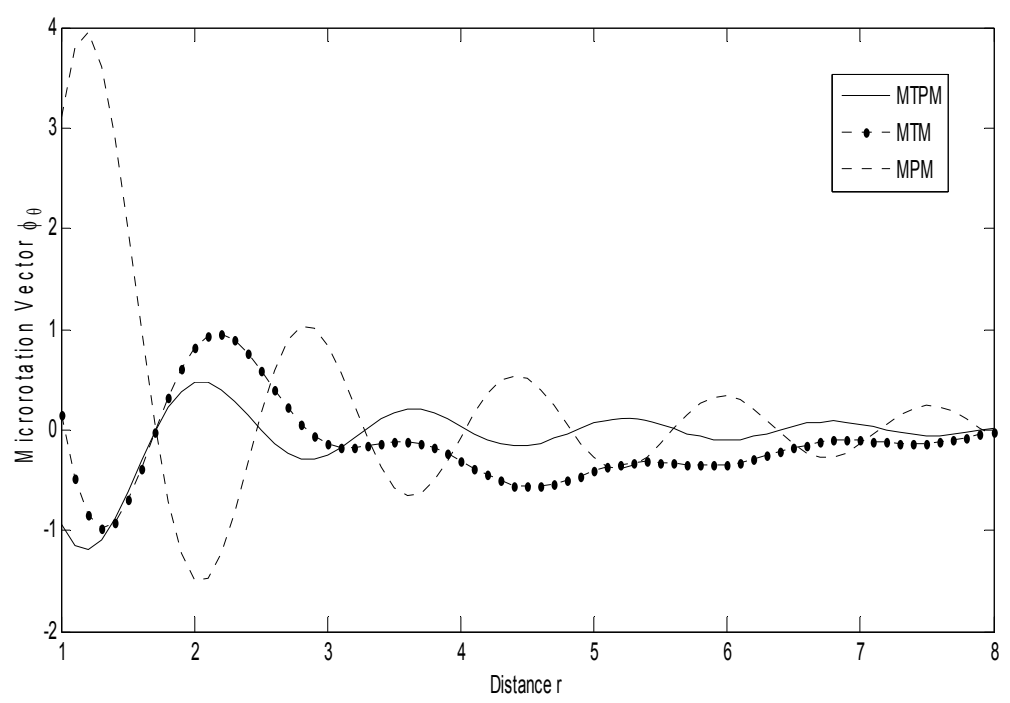

Fig.3. Variations of the microrotation vector $\phi_{\theta}$.

In Fig.3, the value of $\phi_{\theta}$ for MTPM initially decreases for $1 \leq r \leq 1.4$ and oscillates for $1.4 \leq r \leq 8$, whereas for MPM, its value initially increases for $1 \leq r \leq 1.3$, sharply decreases for $1.3 \leq r \leq 2$ and then oscillates for $2 \leq r \leq 8$. For the range $1 \leq r \leq 8$, the behavior of $\phi_{\theta}$ for MTPM and MTM is similar and opposite for MPM.

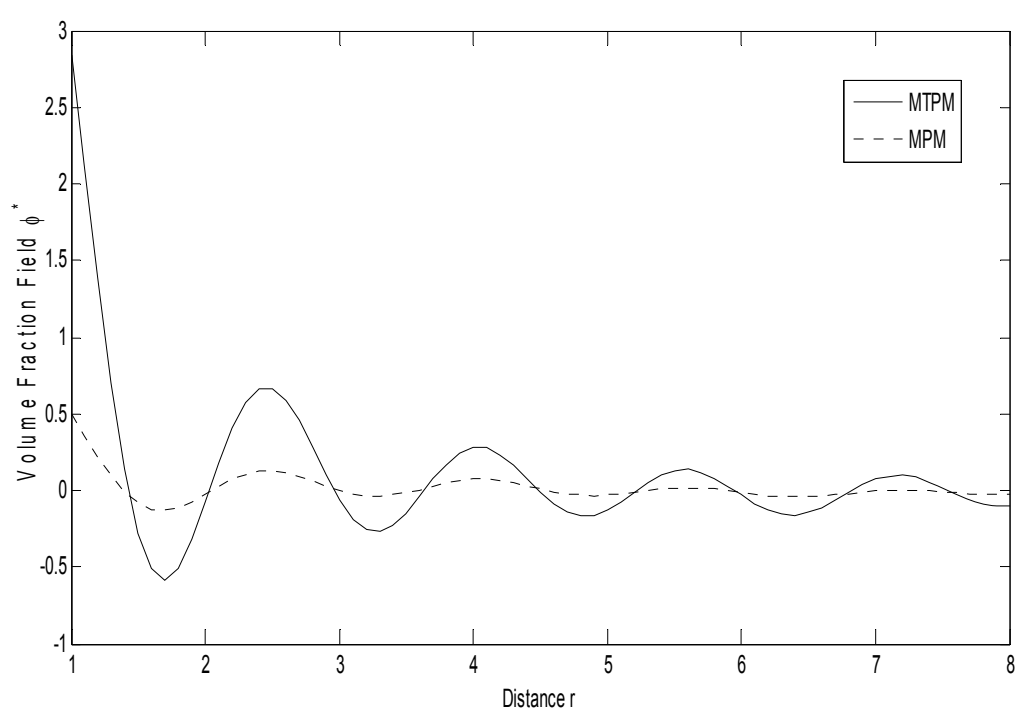

Fig.4. Variations of volume fraction field $\phi^{*}$.

Figure 4 shows that the value of $\phi^{*}$ sharply decreases for $1 \leq r \leq 1.6$ and then oscillates with increasing in amplitude about the origin for MTPM, whereas for MPM, its value decreases for $1 \leq r \leq 1.6$, oscillates for $1.6 \leq r \leq 5.4$ with a large amplitude and further oscillates with a constant amplitude $5.4 \leq r \leq 8$. 


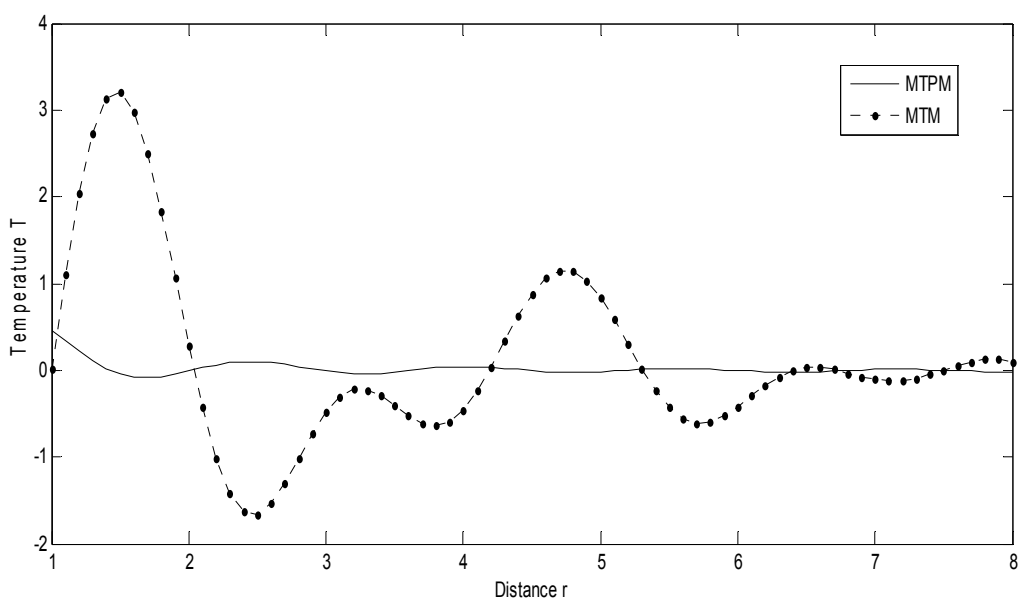

Fig.5. Variations of temperature distribution $T$.

Figure 5 represents that the value of $T$ initially decreases and then oscillates with a large amplitude for the whole range for MTPM. For MTM, its value initially increases for $1 \leq r \leq 1.6$, sharply decreases for $1.6 \leq r \leq 2.5$ and then oscillates for $2.5 \leq r \leq 8$ with different amplitude. The value is large for MTM for $1.2 \leq r \leq 2.2,4.4 \leq r \leq 5.4$ and $7.6 \leq r \leq 8$ and small for $2.2 \leq r \leq 4.4,5.4 \leq r \leq 6.4$ and $6.8 \leq r \leq 7.6$.

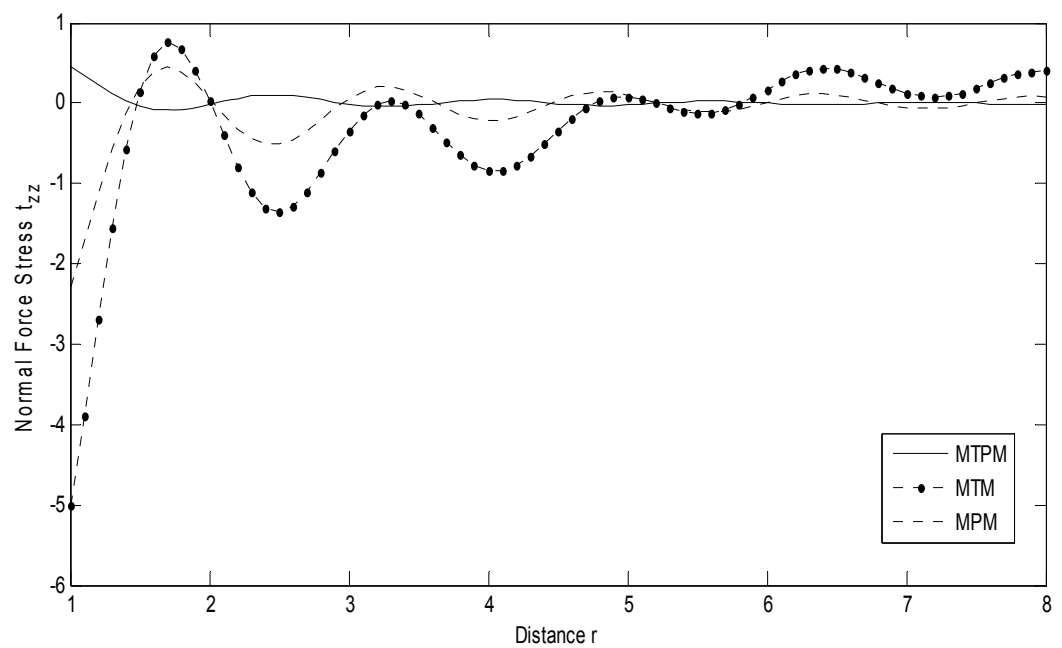

Fig.6. Variations of normal force stress $t_{z z}$.

Figure 6 shows that the value of $t_{z z}$ initially decreases for MTPM for $1 \leq r \leq 1.6$ and then oscillates about the origin with a large amplitude. The value of $t_{z z}$ increases in the beginning for $1 \leq r \leq 1.7$, decreases for $1.7 \leq r \leq 2.6$ and then oscillates for $2.6 \leq r \leq 8$ with $r$ for MTM and MPM. The behavior of $t_{z z}$ for MTPM is opposite to MTM and MPM for $1 \leq r \leq 6.6$. The variation in $t_{z z}$ is oscillatory for MTPM, MTM and MPM. 


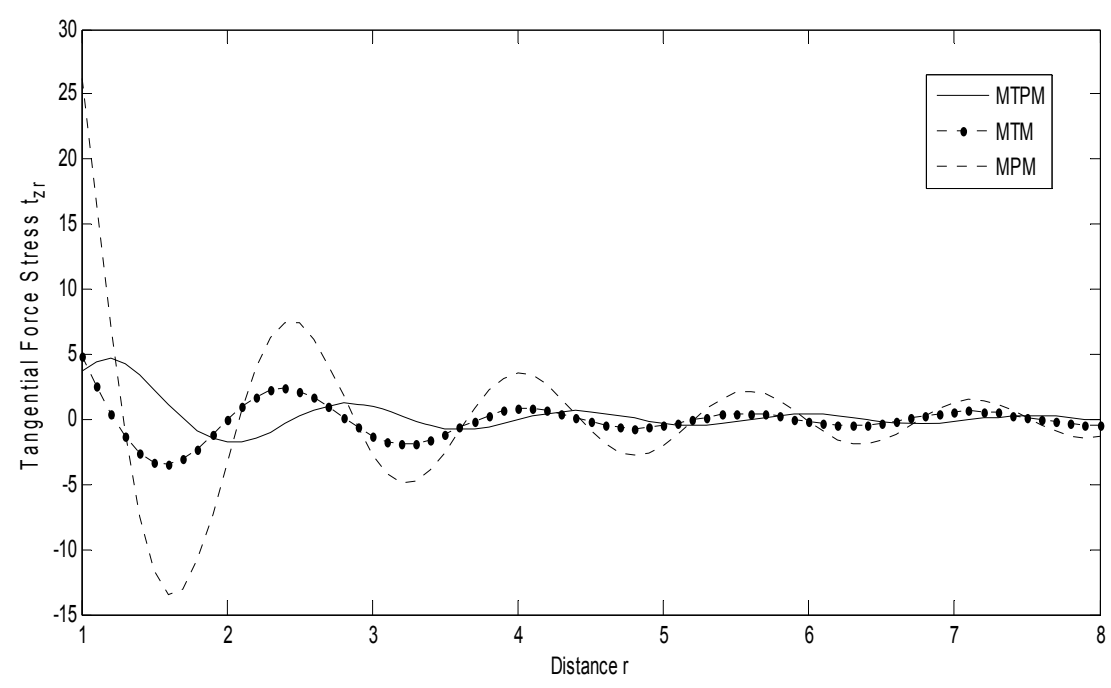

Fig.7. Variation of tangential force stress $t_{z r}$.

Figure 7 shows that for MTPM, the value of $t_{z r}$ increases initially for $1 \leq r \leq 1.4$ and then oscillates with $r$. The value of $t_{z r}$ starts with a sharp decrease for MPM as compared to MTM for $1 \leq r \leq 1.7$ and oscillates about the origin for $1.7 \leq r \leq 8$. The value of $t_{z r}$ for MTPM and MTM is similar near the application of the source and the value is also the same away from the source for the three cases.

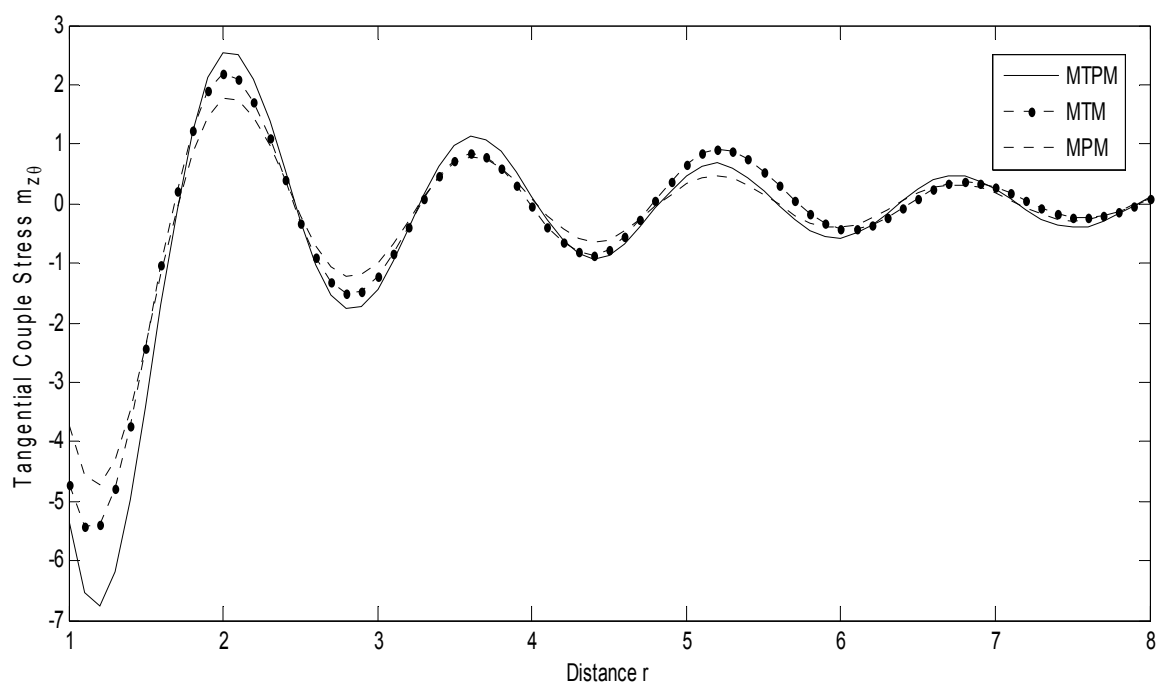

Fig.8. Variations of tangential couple stress $m_{z \theta}$.

Figure 8 shows that the value of $m_{z \theta}$ initially decreases for $1 \leq r \leq 1.4$, sharply increases for $1.4 \leq r \leq 2$ and then oscillates for $2 \leq r \leq 8$ for MTPM, MTM and MPM. The variation and behavior of $m_{z \theta}$ is uniformly oscillatory for the three cases for the whole range. The values for MTPM, MTM and MPM approach zero. 


\section{Conclusions}

The present investigation is concerned with the eigen value approach for a micropolar porous thermoelastic circular plate due to the application of thermo mechanical source. From the figures, it is observed that all the physical quantities have non zero value in considered domain and approache the boundary surface away from the sources. The appreciable effects of the porosity and thermoelasticity are observed on displacements, microrotation, volume fraction field, temperature distribution and stresses. The variation pattern of displacements for MTPM and MPM are the same. The variations patterns are also similar for normal and tangential stresses for MTM and MPM. However, a similar behavior is also observed for tangential couple stress for MTPM, MTM and MPM. The behavior of temperature distribution gets oscillatory due to the porosity effect. Due to the thermal effect, the values of the volume fraction field are initially decreased but afterwards its they increase and oscillate.

\section{Appendix I}

$$
\begin{aligned}
& \lambda_{1}=-\left(a_{11}+a_{22}+a_{33}+a_{44}+a_{55}+b_{12} b_{21}+b_{13} b_{31}+b_{25} b_{52}+b_{24} b_{42}\right), \\
& \lambda_{2}=-a_{14} a_{41}+a_{33} a_{55}+a_{44} a_{55}+a_{11} a_{55}+a_{22} a_{55}+a_{33} a_{44}+a_{11} a_{33}+a_{22} a_{33}+ \\
& +a_{11} a_{44}+a_{22} a_{44}+a_{11} a_{22}-a_{15} a_{51}-a_{45} a_{54}-a_{23} a_{32}+\left(a_{33}+a_{44}+a_{55}\right) b_{12} b_{21}+ \\
& -\left(a_{14} b_{42}+a_{15} b_{52}+a_{32} b_{13}\right) b_{21}+\left(a_{11}+a_{33}+a_{55}\right) b_{42} b_{24}+\left(a_{11}+a_{33}+a_{44}\right) b_{25} b_{52}+ \\
& -\left(a_{41} b_{24}+a_{23} b_{31}+a_{51} b_{25}\right) b_{12}+\left(a_{22}+a_{44}+a_{55}+b_{42} b_{24}+b_{25} b_{52}\right) b_{31} b_{13}+ \\
& -a_{45} b_{52} b_{24}-a_{54} b_{42} b_{25} \text {, } \\
& \lambda_{3}=\left(a_{11} a_{22}+a_{22} a_{55}\right)\left(a_{33}+a_{44}\right)-a_{23} a_{32}\left(a_{11}+a_{44}+a_{55}\right)+ \\
& +a_{11} a_{55}\left(a_{22}+a_{33}+a_{44}\right)+a_{33} a_{44}\left(a_{11}+a_{22}+a_{55}\right)-a_{45} a_{54}\left(a_{11}+a_{22}+a_{33}\right)+ \\
& -a_{14} a_{41}\left(a_{22}+a_{33}+a_{55}\right)-a_{15} a_{51}\left(a_{22}+a_{33}+a_{44}\right)+ \\
& +b_{42} b_{25}\left(a_{14} a_{51}+a_{11} a_{54}+a_{33} a_{54}\right)+b_{52} b_{25}\left(-a_{14} a_{41}+a_{11} a_{33}+a_{14} a_{44}+a_{33} a_{44}\right)+ \\
& +b_{52} b_{24}\left(a_{15} a_{41}-a_{11} a_{45}+a_{33} a_{45}\right)-b_{12} b_{25}\left(a_{33} a_{51}+a_{44} a_{51}-a_{41} a_{54}\right)+ \\
& -b_{12} b_{24}\left(a_{33} a_{41}-a_{45} a_{51}+a_{41} a_{55}\right)+b_{42} b_{24}\left(-a_{15} a_{51}+a_{11} a_{33}+a_{11} a_{55}+a_{33} a_{55}\right)+ \\
& -a_{32} b_{21} b_{13}\left(a_{44}+a_{55}\right)+b_{31} b_{13}\left(a_{22} a_{44}+a_{22} a_{55}+a_{44} a_{55}-a_{45} a_{54}\right)+ \\
& +b_{21} b_{42}\left(a_{15} a_{54}-a_{14} a_{33}-a_{14} a_{55}\right)+b_{21} b_{52}\left(-a_{14} a_{45}-a_{15} a_{33}-a_{15} a_{44}\right)+ \\
& +b_{12} b_{21}\left(-a_{45} a_{54}+a_{33} a_{44}+a_{44} a_{55}+a_{33} a_{55}\right)-b_{12} b_{31}\left(a_{23} a_{44}+a_{23} a_{55}\right)+ \\
& -b_{31} b_{13}\left(a_{54} b_{42} b_{25}-a_{44} b_{52} b_{25}+a_{45} b_{52} b_{24}\right)+a_{32} b_{13}\left(a_{51} b_{25}+a_{41} b_{24}\right)+ \\
& +a_{15} a_{41} a_{54}+a_{14} a_{45} a_{51}+a_{14} a_{23} b_{31} b_{42} \text {, }
\end{aligned}
$$




$$
\begin{aligned}
& \lambda_{4}=\left(a_{44} a_{55}-a_{45} a_{54}\right)\left(a_{23} b_{12} b_{31}-a_{33} b_{12} b_{21}\right)+ \\
& +\left(a_{15} a_{54}-a_{14} a_{55}\right)\left(a_{33} b_{21} b_{42}-a_{23} b_{31} b_{42}\right)+\left(a_{14} a_{45}-a_{15} a_{44}\right)\left(a_{33} b_{21} b_{52}-a_{23} b_{31} b_{52}\right)+ \\
& +\left(a_{45} a_{54}-a_{44} a_{55}\right)\left(a_{32} b_{21} b_{13}-a_{22} b_{31} b_{13}\right)+a_{33} b_{12} b_{24}\left(a_{45} a_{51}+a_{33} a_{41} a_{55}\right)+ \\
& +a_{33} b_{42} b_{24}\left(-a_{15} a_{51}+a_{11} a_{55}\right)+a_{32} b_{13} b_{24}\left(a_{41} a_{55}-a_{45} a_{51}\right)+ \\
& +\left(a_{41} a_{54}-a_{44} a_{51}\right)\left(a_{33} b_{12} b_{25}-a_{32} b_{13} b_{25}\right)+a_{33} b_{42} b_{25}\left(a_{14} a_{51}-a_{11} a_{54}\right)+ \\
& +a_{33} b_{52} b_{25}\left(a_{11} a_{44}-a_{14} a_{41}\right)+a_{15} a_{51}\left(a_{23} a_{32}-a_{22} a_{33}-a_{22} a_{44}-a_{33} a_{44}\right)+ \\
& +a_{14} a_{45} a_{51}\left(a_{22}+a_{33}\right)+a_{15} a_{41} a_{54}\left(a_{22}+a_{33}\right)+a_{45} a_{54}\left(a_{23} a_{32}-a_{11} a_{22}-a_{11} a_{33}+\right. \\
& \left.-a_{22} a_{33}\right)+a_{14} a_{41}\left(a_{23} a_{32}-a_{22} a_{33}-a_{22} a_{55}-a_{33} a_{51}\right)-a_{11} a_{23} a_{32}\left(a_{44}+a_{55}\right)+ \\
& +a_{55}\left(a_{11} a_{22} a_{33}-a_{23} a_{32} a_{44}\right)+a_{11} a_{22} a_{44}\left(a_{33}+a_{55}\right)+a_{33} a_{44} a_{55}\left(a_{11}+a_{22}\right)+ \\
& +\left(a_{15} a_{41}-a_{11} a_{45}\right) a_{33} b_{24} b_{52}, \\
& \lambda_{5}=\left(a_{22} a_{33}-a_{23} a_{32}\right)\left(a_{11} a_{44} a_{55}-a_{11} a_{45} a_{54}+a_{14} a_{45} a_{51}\right)+ \\
& +\left(a_{15} a_{54}-a_{14} a_{55}\right)\left(a_{22} a_{33} a_{41}-a_{23} a_{32} a_{41}\right)+\left(a_{32}+a_{33}\right) a_{15} a_{23} a_{44} a_{51} .
\end{aligned}
$$

\section{Appendix II}

$$
\begin{aligned}
& a_{i}=\frac{\xi}{\Delta_{i}}\left[r_{1}^{2}\left\{r_{2}\left(r_{3}\left(1-\delta^{2}\right)+p \delta^{* 2}\right)-p_{0}^{2} \delta_{1}^{*} r_{3}\right\}+\right. \\
& \left.+\epsilon r_{1} r_{5}\left\{r_{3}\left(r_{2}-\bar{v}^{2} \delta_{l}^{*}\left(1-\delta^{2}\right)-2 \bar{v} p_{0} \delta_{l}^{*}-\bar{v} \delta_{l}^{*}\right)+p \bar{v}^{2} \delta_{l}^{*} \delta^{* 2}\right\}\right] \\
& b_{i}=\frac{-1}{\Delta_{i}}\left[r_{I}^{2}\left\{r_{2}\left(r_{3} r_{4}+p \delta^{* 2} q_{i}^{2}\right)-p_{0}^{2} \delta_{1}^{*} \xi^{2} r_{3}\right\}+\right. \\
& \left.+\epsilon r_{I} r_{3} r_{5}\left(\xi^{2} r_{2}+\bar{v}^{2} \delta_{1}^{*} r_{4}-2 \bar{v} p_{0} \delta_{1}^{*} \xi^{2}\right)+\epsilon p \bar{v}^{2} \delta_{1}^{*} \delta^{* 2} q_{i}^{2} r_{1} r_{5}\right] \\
& d_{i}=\delta_{1}^{*}\left(p_{0} r_{1}+\epsilon \overline{\mathrm{v}} r_{5}\right)\left(\xi a_{i}+b_{i}\right) q_{i} /\left(-r_{1} r_{2}-\epsilon \overline{\mathrm{v}}^{2} \delta_{1}^{*} r_{5}\right), \\
& e_{i}=\epsilon\left(s+\eta_{0} \tau_{0} s^{2}\right)\left\{\left(r_{I} r_{2}+\epsilon \bar{v}^{2} \delta_{1}^{*} r_{5}\right)-\epsilon \bar{v} \delta_{I}^{*}\left(p_{0} r_{I}+\epsilon \bar{v} r_{5}\right)\right\}\left(\xi a_{i}+b_{i}\right) q_{i} /\left\{r_{l}\left(-r_{1} r_{2}-\epsilon \bar{v}^{2} \delta_{1}^{*} r_{5}\right)\right\} \text {, } \\
& \Delta_{i}=\delta^{* 2}\left[r_{1}^{2}\left\{r_{2}\left(\xi^{2}+s^{2}-q_{i}^{2}\right)+p_{0}^{2} \delta_{1}^{*}\left(q_{i}^{2}-\xi^{2}\right)\right\}+\right. \\
& \left.+\epsilon r_{1} r_{2} r_{5}\left(\xi^{2}-q_{i}^{2}\right)+\epsilon \delta_{1}^{*} r_{1} r_{5}\left\{\bar{v}^{2}\left(\xi^{2}+s^{2}-q_{i}^{2}\right)-2 p_{0} \bar{v}\left(\xi^{2}-q_{i}^{2}\right)\right\}\right], \\
& r_{1}=\left(\xi^{2}+Q^{*}\left(s+\tau_{0} s^{2}\right)-q_{i}^{2}\right), \quad r_{2}=\left(\xi^{2}+\delta_{3}^{*} s^{2}+p_{I} \delta_{1}^{*}+\delta_{2}^{*} s-q_{i}^{2}\right), \\
& r_{3}=\left(\xi^{2}+\frac{s^{2}}{\delta_{1}^{2}}+2 \delta^{* 2}-q_{i}^{2}\right), \quad r_{4}=\left(\xi^{2}+s^{2}-\delta^{2} q_{i}^{2}\right), \quad r_{5}=\left(1+\tau_{1} s\right)\left(s+\eta_{0} \tau_{0} s^{2}\right) .
\end{aligned}
$$




\section{Nomenclature}

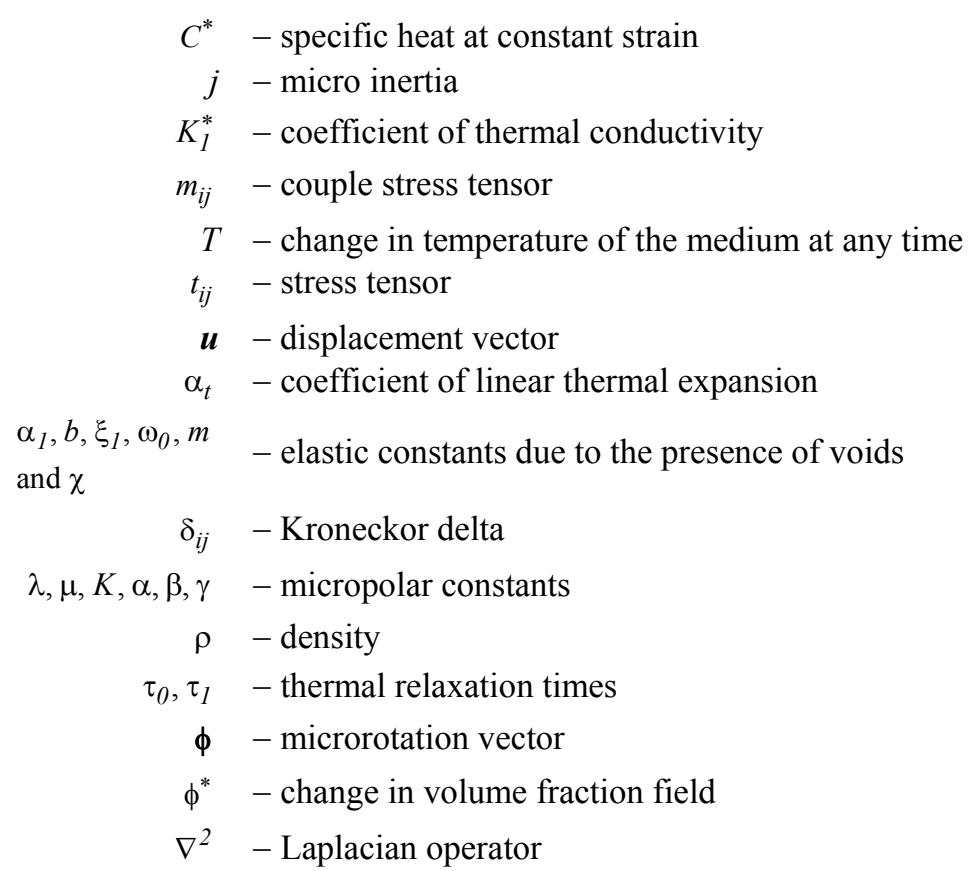

\section{References}

[1] Eringen A.C. (1966): Linear theory of micropolar elasticity. - Journal of Mathematics and Mechanics, vol.15, pp.909-923.

[2] Gauthier R.D. (1982): Experimental investigation on micropolar media. - Mechanics of Micropolar Media, CSIM Courses and Lectures, vol.1, pp.395-463.

[3] Eringen A.C. (1970): Foundations of Micropolar Thermoelasticit. - CSIM Udine, Course of Lectures 23, CDROM.

[4] Nowacki M. (1966): Couple-stresses in the theory of thermoelasticity. - Proceedings. IUTAM Symposia, vol.1, pp.259-278.

[5] Tauchert T.R., Claus Jr. W.D. and Ariman T. (1968): The linear theory of micropolar thermoelasticity, International Journal of Engineering Science, vol.6, pp.36-47.

[6] Boschi E. and Iesan D. (1973): A generalized theory of linear micropolar thermoelasticity. - Mechanica, vol.8, pp.154-157.

[7] Passarella F. (1996): Some results in micropolar thermoelasticity. - Mechanics Research Communications, vol.23, pp.349-357.

[8] Cowin S.C. and Nunziato J.W. (1983): Linear elastic materials with voids. - Journal of Elasticity, vol.13, pp.125147.

[9] Iesan D. (1985): Shock waves in micropolar elastic materials with voids. - Analele Stiintificeli Universita atti.I. Cuza, din Iase, vol.31, pp.177-186.

[10] Iesan D. (1986): A theory of thermoelastic materials with voids. - Acta Mechanica, vol.60, pp.67-89.

[11] Scarpetta E. (1990): On the fundamental solutions in micropolar elasticity with voids. - Acta Mechanica, vol.82, pp.151-158. 
[12] Marin M. (1995): The mixed problem in elastostatic of micropolar materials with voids. - An: Stiinf Uni. Ovidius Constanta Ser. Mat., vol.3, pp.106-117.

[13] Marin M. (1996): Generalized solutions in elasticity of micropolar bodies with voids. - Rev. Acad. Canaria. Cienc., vol.8, pp.101-106.

[14] Ciarletta M., Scalia A. and Svanadze (2007): Fundamental solution in the theory of micropolar thermoelasticity for materials with voids. - Journal of Thermal Stresses, vol.30, No.3, pp.213-229.

[15] Kumar R. and Panchal M. (2011): Study of Circular Crested Waves in Micropolar Porous Medium Possessing Cubic Symmetry. - Bulletin of the Polish Academy of Sciences, Technical Sciences, vol.59, No.1.

[16] Ailawalia P. and Kumar R. (2011): Thermomechanical deformation in microplar porous thermoelastic material. Mechanics of Advanced Materials and Structures, vol.18, No.4, pp.255-261.

[17] Othman M.I.A. and Youssef Atwa S (2012): Response of micropolar thermoelastic solid with voids due to various sources under green-naghdi theory. - Acta Mechanica Solida Sinica, vol.25, No.2, pp.197-209.

[18] Sharma K. and Marin M. (2013): Effect of Distinct Conductive and Thermodynamic Temperatures on the Reflection of Plane Waves in Micropolar Elastic Half-Space. - U.P.B. Sci. Bull. Series A, vol.75, No.2.

[19] Sharma K., Sharma S. and Bhargava R.R. (2013): Propagation of waves in micropolar thermoelastic solid with two temperatures bordered with layers or half spaces of inviscid liquid. - Materials Physics and Mechanics, vol.16, pp.64-81.

[20] Sharma K. (2013): Reflection at free surface in micropolar thermoelastic solid with two temperatures. International Journal of Applied Mechanics and Engineering, vol.18, No.1, pp.217-234.

[21] Sharma K. and Kumar P. (2013): Propagation of plane waves and fundamental solution in thermoviscoelastic medium with voids. - Journal of Thermal Stresses, vol.36, pp.94-111.

[22] Kumar R., Sharma K.D. and Garg S.K. (2015): Fundamental solution in micropolar viscothermoelastic solids with void. - International Journal of Applied Mechanics and Engineering, vol.20, No.1, pp.109-125.

[23] Marin M. (2016): An approach of a heat flux dependent theory for micropolar porous media. - Mechanica, vol.51, No.5, pp.1127-1133.

[24] Kumar R. and Partap G. (2008): Porosity effect on circular crested waves in micropolar thermoelastic homogeneous isotropic plate. - International Journal of Applied Mathematics and Mechanics, vol.4, No.2, pp.118.

[25] Eringen A.C. (1984): Plane waves in non-local micropolar elasticity. - International Journal of Engineering Science, vol.22, pp.1113-1121.

[26] Dhaliwal R.S. and Singh A. (1980): Dynamical Coupled Thermoelasticity. - New Delhi: Hindustan Publication Corporation.

Received: October 6, 2016

Revised: May 29, 2017 